\title{
Longitudinal Sleep Efficiency in the Elderly and Its Association with Health
}

DOI:

$10.1111 /$ jsr. 12898

\section{Document Version}

Accepted author manuscript

Link to publication record in Manchester Research Explorer

\section{Citation for published version (APA):}

Didikoglu, A., Maharani, A., Tampubolon, G., Canal, M., Payton, A., \& Pendleton, N. (2019). Longitudinal Sleep Efficiency in the Elderly and Its Association with Health. Journal of Sleep Research.

https://doi.org/10.1111/jsr.12898

\section{Published in:}

Journal of Sleep Research

\section{Citing this paper}

Please note that where the full-text provided on Manchester Research Explorer is the Author Accepted Manuscript or Proof version this may differ from the final Published version. If citing, it is advised that you check and use the publisher's definitive version.

\section{General rights}

Copyright and moral rights for the publications made accessible in the Research Explorer are retained by the authors and/or other copyright owners and it is a condition of accessing publications that users recognise and abide by the legal requirements associated with these rights.

\section{Takedown policy}

If you believe that this document breaches copyright please refer to the University of Manchester's Takedown Procedures [http://man.ac.uk/04Y6Bo] or contact uml.scholarlycommunications@manchester.ac.uk providing relevant details, so we can investigate your claim.

\section{OPEN ACCESS}




\section{Title Page}

\section{Title:}

Longitudinal Sleep Efficiency in the Elderly and Its Association with Health

\section{Running Title:}

Ageing and Sleep Efficiency

\section{Authors and author affiliations:}

Altug Didikoglu (1), Asri Maharani (1), Gindo Tampubolon (2), Maria Merce Canal (1), Antony Payton (3), Neil Pendleton (1)

1) Division of Neuroscience \& Experimental Psychology, The University of Manchester, UK

2) School of Environment, Education and Development, The University of Manchester, UK

3) Division of Informatics, Imaging \& Data Sciences, The University of Manchester, UK The institution where the work was performed: (1)

\section{Corresponding author's full address and corresponding author's current email:}

Name:

Altug Didikoglu

Address:

Division of Neuroscience \& Experimental Psychology, The University of Manchester, AV Hill Building, Upper Brook Street, M13 9PT, Manchester, UK

\section{Email:}

altug.didikoglu@postgrad.manchester.ac.uk 


\section{Author contribution:}

A.D performed the data analysis, drafted the manuscript and designed the figures. A.M., G.T., M.C., A.P. and N.P were involved in planning and supervised the work. A.P. and N.P. own the data of the cohort. All authors discussed the results and commented on the manuscript.

\section{Funding}

The University of Manchester Longitudinal Study of Cognition in Normal Healthy Old Age cohort has been supported by a series of grant funders including Medical Research Council, Economic and Social Research Council, Biotechnology and Biological Sciences Research Council, Wellcome Trust and Age UK. Altug Didikoglu was supported by a grant from the Republic of Turkey Ministry of National Education.

\section{Conflicts of interest}

All authors declare no conflicts of interest. 


\section{Abstract}

The relationships between older age and sleep efficiency have traditionally been assessed using cross-sectional studies that ignore changes within individuals as they age. This research examines the determinants of sleep efficiency, the heterogeneity in individual's sleep efficiency trajectory across a period of up to 27 years in later life and its associations with health. The University of Manchester Longitudinal Study of Cognition in Normal Healthy Old Age cohort ( $\mathrm{N}=6375,42-94$ age) was used in this study. Depression and health data was collected using self-report validated instruments (Cornell Medical Index, Beck Depression Inventory, Geriatric Depression Scale). Longitudinal sleep and socio-demographics data was collected using a study-specific self-report questionnaire. Mixed-effect model was performed for sleep efficiency with adjustments for time-invariant and time-variant predictors. Latent class analysis was used to demonstrate subgroups of sleep efficiency trajectories and associations between sleep efficiency clusters and health history of the participants were investigated. Older adults have decreased sleep efficiency over time with $18.6 \%$ decline between 40 and 100 years of age. Three sleep efficiency trajectory clusters were identified: high (32\%), medium (50\%), and low sleep efficiency (18\%). Belonging to the high sleep efficiency cluster was associated with having lower prevalence of hypertension, circulatory problems, general arthritis, breathing problems and recurrent episodes of depression compared to the low efficiency cluster. Overall, ageing decreases sleep efficiency. However, there are detectable subgroups of sleep efficiency that are related to different disease prevalence.

\section{Keywords}

older adults, ageing, sleep efficiency, sleep trajectory, latent class analysis, physical and mental health 


\section{INTRODUCTION}

Sleep is a conserved characteristic from invertebrates to mammals and there is strong evidence that it is responsible for metabolic homeostasis, memory consolidation and detoxification of the brain(Anafi, Kayser, \& Raizen, 2019; Xie et al., 2013). Sleep quality is an important contributor towards public health with sleep problems being associated with cardiovascular diseases, metabolic syndrome, diabetes, mood disorders, neurodegenerative disorders, cancer and mortality(Kripke, Garfinkel, Wingard, Klauber, \& Marler, 2002; Lucey et al., 2019; Medic, Wille, \& Hemels, 2017). Understanding how sleep is regulated and influenced by other factors is necessary to improve sleep health. Even though both timing and duration are more than $40 \%$ heritable,(Dashti et al., 2019; Lane et al., 2017) social, environmental and other biological factors contribute to sleep architecture. Older age is one of the best predictors of change in sleep structure. A meta-analysis combining data from 65 studies, which investigated the effects of ageing on sleep using polysomnography or actigraphy reported that with each decade, sleep duration and sleep efficiency decrease by 10 minutes and $3 \%$ respectively(Ohayon, Carskadon, Guilleminault, \& Vitiello, 2004). This study also showed that sleep latency and awakenings during sleep increase with ageing, however, the amount of REM (rapid eye movement) sleep and to an even greater extent, deep sleep (slow wave sleep), decrease(Ohayon et al., 2004). In addition, ageing has been characterized with earlier sleep timing and excessive daytime sleepiness that induces daytime napping(Mander, Winer, \& Walker, 2017).

Sleep problems have been investigated in terms of their different dimensions such as timing, duration, disruption or efficiency. Among them, only sleep efficiency has been indicated to continuously decline in older ages (>60 years)(Ohayon et al., 2004). Clustering of poor sleepers has shown that inefficient sleepers are mostly elderly people(Gadie, Shafto, Leng, Cam-CAN, \& Kievit, 2017). Therefore, negative health consequences of low sleep efficiency that covary with ageing in later life require further investigation. However, ageing and sleep studies have commonly been performed with cross-sectional data, which do not consider the 
heterogeneity of the populations and the variations of each individual across ages. There are few studies investigating longitudinal sleep change in the elderly but their follow-up periods (<10 years) and sample sizes are limited (<150 participants)(Bliwise, Ansari, Straight, \& Parker, 2005; Hoch et al., 1997). Here we use longitudinal sleep and health data from the University of Manchester Longitudinal Study of Cognition in Normal Healthy Old Age (UMLCHA) cohort to investigate individual sleep trajectories and their associations with physical and mental health. We firstly aimed to identify how sleep efficiency was associated with chronological age and sociodemographic factors considering longitudinal individual variations. We then identified unobserved heterogeneity of sleep efficiency using latent class analysis. Subgroups of longitudinal sleep efficiency changes were then evaluated for their health.

\section{METHODS}

\subsection{Population}

The UMLCHA cohort recruited 6375 participants in Greater Manchester and Newcastle upon Tyne between 1982-1994(Rabbitt et al., 2004). We consider a wave to be the period including the distribution of the questionnaire for each specific questionnaire. Since recruitment of the cohort was a 10-year period, each participant attended the surveys at different dates, therefore, waves were overlapping (Figure 1). The mean age for the baseline was $65.19(\mathrm{SD}=7.45)$ years (Table 1). The cohort comprised of $69.9 \%$ female participants. Individuals commonly had intermediate occupations and attained lower secondary education. More than $84 \%$ of participants were retired, housewife/husband or unemployed. In each waves, at least $90 \%$ of the respondents rated themselves as having fair or good health.

\subsection{Questionnaires}

Participants provided a self-report of their demographics, subjective health, lifestyle including sleep characteristics (Personal Details Questionnaire) and mood (Beck Depression Inventory, Geriatric Depression Scale 30-item and 15-item(Alden, Austin, \& Sturgeon, 1989; 
Beck, Steer, \& Carbin, 1988). The Personal Details Questionnaire (PDQ) was assessed in 5 waves between 1982 and 2010 (maximum follow-up period 27.5 years) (Figure 1). Depression was assessed every 2-3 years using Beck Depression Inventory (BDI) in the first 2 waves, Yesavage Geriatric Depression Scale (GDS) 30-item long version in 4 waves or a 15-item short version in the final 5 waves ( $N=5490$, between 1982 and 2017) (Table S1). The Cornell Medical Index (CMI)(Brodman, Erdmann, Lorge, Wolff, \& Broadbent, 1949) was administrated in 4 waves to examine symptoms of physical and mental diseases $(N=2713$, between 1989 and 2007) (see supplementary materials for details of longitudinal test dates). Written informed consent was obtained from all respondents at onset of the study. All self-report and questionnaire data were collected under the approval of The University of Manchester research ethics committee. This paper includes the secondary data analysis of the anonymized data set.

\subsubsection{Predictors}

Time-invariant and time-variant predictors were collected using the PDQ. Baseline information included birth date, sex (male/female), economic status (Standard occupational classification 2000-6 levels) and educational attainment (International Standard Classification of Education 1997-7 levels) categories. In each wave, their current working status (yes/no), alcohol intake (no drinking/several alcohol-free days each week/consuming every day) and smoking (yes/no), subjective health rating (very good to very bad-5 levels), marital status (single/married or living with someone) and usage of sleep medication (yes/no) were repeatedly recorded. Age (years) of participants were calculated by subtracting birth date from test date.

\subsubsection{Sleep measures}

PDQ also contains a study-specific sleep diary in which participants were asked to provide getting up ("Generally at what time do you get up in the morning?") and going to bed times ("Generally at what time do you go to bed at night?") along with their sleep duration ("On 
average how many hours sleep do you get every night?"). Sleep fragmentation was also asked as number of night wakes ("How many times during the night do you wake up?"). Sleep efficiency (\%) was calculated as "sleep duration*100/(getting up time - going to bed time)". Sleep efficiency above $100 \%$ was accepted as $100 \%$.

\subsubsection{Health-related variables}

Since three different depression inventories were used, depression was coded as normal or mild to severe categorical form for all waves. For BDI, the maximum total score was 63 with scores greater than 9 treated as depressed(Beck, Steer, \& Carbin, 1988). For GDS, the long form total score was 30 and the short form 15. Individuals classified as depressed were scored above 11 and 5 respectively(Alden et al., 1989). Depression was grouped according to reoccurrence of depression if participants attended at least two surveys. Recurrent depression was defined as more than one episode of above-threshold score on a validated questionnaire. For CMI, each "yes" answer (out of 201 for women/208 for men) was counted as a symptom of a disorder or disease. An Individual was considered having a health problem if there was at least one 'yes' answer to the specific question that refers to the patient's medical history of this specific condition in 4 waves. With this symptomology, hypertension, heart attack, circulatory problems, arthritis, epilepsy, stroke, Parkinson`s disease, diabetes, cancer and breathing problems were coded categorical (yes/no). Comorbidity was calculated to be the total number of health problems in the CMI. Comorbidities were categorized as no comorbidity, having one health problem or having more than one health problems.

\subsection{Statistical analysis}

Descriptive data were presented as mean \pm standard deviation (SD) or as the frequency of different groups. The analyses were performed in three different steps. Firstly, change of sleep efficiency across ages was investigated using mixed-effect linear model. The mixed model includes both fixed and random effects, which enables the search for population 
trajectories over waves accounting for within individual variations. Missing data due to dropout was assumed missing at random. Time-independent variables consisted of sex, level of education and social class. Time-dependent factors comprised age, subjective health rating, marital status, working status, smoking, drinking and usage of sleep medication. The analysis was performed using Stata Statistical Software: Release 14. College Station, TX: StataCorp LP. Regression results were shown as coefficient (B) \pm standard error (SE).

Secondly, latent class analysis was performed for sleep efficiency variable to detect subgroups with distinct trajectories. This analysis was undertaken using LatentGOLD 5.1. Belmont, MA: Statistical Innovations. The same time-dependent and time-independent predictors were included in the analyses as covariates. A 3-cluster model was found to have the best fit compared to other models with up to 5 classes according to these criteria: Lower Bayesian Information Criterion (BIC), the mean likelihood of belonging to a cluster being more than $70 \%$, sample size for each cluster having at least 0.5 of all individuals and the distinction between clusters(Lassale et al., 2019) (Table S3). Sleep duration, getting up time, going to bed time and number of night awakenings were compared between detected sleep efficiency latent classes to figure out which sleep parameter estimated clustering best.

Finally, the risk of presence of diseases detected with the $\mathrm{CMI}$ and depression inventories were compared between obtained sleep efficiency latent classes. Pearson chi-square $\left(x^{2}\right)$ test was performed to investigate associations. The threshold for significant $p$-value was 0.05 .

\section{RESULTS}

\subsection{Description of cohort characteristics}

Data in the first wave showed that individuals went to bed on average at 23:15 (SD=48 minutes) (hh:mm) and got up at 7:51 ( $\mathrm{SD}=47$ minutes) (Table 2). Their average reported sleep duration was $6.97(\mathrm{SD}=1.18)$ hours and the mean sleep efficiency was $81.45 \%$ $(S D=13.31) .3387$ participants $(62.2 \%)$ reported $80 \%$ or more sleep efficiency, in which 468 
participants (8.6\%) had $100 \%$ sleep efficiency. The average number of waking during the night was $1.37(S D=1.24)$, in which $31.8 \%$ of participants reported no night waking.

2898 participants (45.5\%) attended only the first wave. $54.5 \%$ of the cohort had available data for 2 or more PDQ waves. Total number of participants completing the PDQ twice is 3477 , three times is 873 , four times is 564 and all 5 times is 212 . Average sleep duration and efficiency decreased to $6.74(S D=1.29)$ hours and $75.70 \%(S D=13.73)$ in the last wave. Average sleep fragmentation increased by 0.86 between first and last measures, 2.23 $(S D=1.03)$ in the last survey. The mean of going to bed times shifted towards earlier points during this period, however, the mean of getting up time showed minimal change. Therefore, the mean of total time in bed increased with ageing.

\subsection{Effects of ageing and socio-demographics on sleep efficiency}

Mixed model analysis showed significant decrease in sleep efficiency with age, by $3.1 \%$ per decade $(B=-0.31, S E=0.01, p<0.001)$ (Figure 2). In the estimated model, mean sleep efficiency was $89.8 \%(S D=5.35)$ before age 50 . With increasing age this declined, with those aged between $60-80$ years having a mean sleep efficiency $79.9 \%(S D=4.15)$ and those aged over 90 years having a mean sleep efficiency of approximately $71.8 \%(S D=3.53)$.

In the longitudinal statistical model, subjective health rating and socio-demographic features of the individuals were also included (Table 3). We observed that sleep efficiency of females was $5 \%$ less than males $(B=-5.02, S E=0.40, p<0.001)$. Social class had no effect on sleep efficiency. However, individuals with post-secondary non-tertiary education or tertiary education first stage had higher sleep efficiency compared to those with lower education levels. Employed people's sleep efficiency was $2 \%$ higher $(B=1.67, S E=0.36, p<0.001)$ than retired, housewife or unemployed participants. Living with a partner did not affect sleep efficiency. The participants whose general health rating was very good had more efficient sleep $(B=5.76, S E=1.95, p=0.003)$ than those whose general health rating was very bad. Interestingly, non-smokers had lower sleep efficiency $(B=-1.97, \quad S E=0.40, p<0.001)$. 
Consuming alcohol up to once a day was associated with lower sleep efficiency $(B=-0.80$, $\mathrm{SE}=0.31, \mathrm{p}=0.01)$ than consuming no alcohol. Sleep efficiency of individuals not using sleep medication was significantly higher than those using sleep medication $(B=4.99, S E=0.45$, $p<0.001)$. The results were controlled for their robustness using a joint model to deal with the attrition in the longitudinal design(Tampubolon \& Maharani, 2018). The mixed model results were compared with those of attrition-corrected model and similarity between the results supported the random drop-outs (Table S2).

\subsection{Sleep efficiency latent classes}

Within individual variation of sleep efficiency across age may not be the same for all people in the cohort. To explore this, we performed latent class analysis to investigate for unobserved clusters within the sleep efficiency trajectories. As a hypothesis-free method, we prespecified a limit of up to 5 classes. Sleep efficiency trajectories were divided into three subgroups that gave the best fit (Figure 3). The effect of ageing significantly differed between these subgroups ( $\mathrm{Wald}(2)=103.58, \mathrm{p}<0.001)$ (Table S4). The first cluster had almost $95 \%$ sleep efficiency at age 50 , hence this subgroup was called the high sleep efficiency cluster (32\% of the cohort). Their sleep efficiency was largely time-invariant, as shown by having more than $85 \%$ sleep efficiency in their 90 s $(B=-0.16)$. The second $(B=-$ 0.40) and the third $(B=-0.38)$ sleep efficiency clusters were more variable than the high efficiency group. The second group had approximately $85 \%$ sleep efficiency (the medium sleep efficiency cluster, $50 \%$ of the cohort) at age 50 while the third group (the low sleep efficiency cluster, $18 \%$ of the cohort) had $65 \%$. The second and third clusters had more than $15 \%$ decrease in sleep efficiency from age 40 to $90+$ years. Variances of clusters also significantly differed from each other $(\operatorname{Wald}(2)=465.80, p<0.001)$, in which the low efficiency cluster had the highest variance (139.63) and the high efficiency cluster had the lowest variance (35.35). Therefore, the clustering has been driven by both baseline sleep efficiency and its longitudinal variation (Figure S1). To investigate whether the clustering affected by individuals whose longitudinal data was missing, a sensitivity analysis was performed. Since 
clusters produced only with individuals who had longitudinal (at least 2 waves) sleep efficiency data $(\mathrm{N}=3019)$ were similar to the original results, we considered our results robust (Figure S2).

To characterize why sleep efficiency differed between these latent classes, we demonstrated how sleep duration, number of night wakes and sleep timing of each class change across ages (Figure 4). The high efficiency cluster preserved their sleep duration across age compared to medium or low efficiency clusters that had more than 1-hour decline in their sleep duration (Figure 4A). The number of sleep fragmentations was higher in the low efficiency cluster and decreased in the medium and the high clusters. However, the slope of the increase in the number of night wakes was steeper in the high efficiency cluster compared to the low efficiency cluster (Figure 4B). Getting up time was similar across clusters and not time-variant (Figure 4C). In contrast, going to bed time was later in the high efficiency cluster but the change across age was parallel for all clusters (Figure 4D).

\subsection{Depression}

The mean BDI score for the cohort was $7.79(S D=6.44)$ and prevalence of mild to severe depression was $29.5 \%$ at the baseline. During the study period (11 depression surveys), $57 \%$ of the cohort did not experience depression at any time. The frequency of being depressed once during the whole study period was $20 \%$. The remaining $23 \%$ consisted of those who reported recurrent depression. The risk of recurrence was $24 \%$ higher in low efficiency cluster compared to high efficiency cluster $\left(\mathrm{Chi}^{2}(2)=135.43, \mathrm{p}<0.001\right)$ (Table 4).

\subsection{Cornell Medical Index}

The CMI was surveyed 4 times during the study period and included questions covering 10 chronic problems. Breathing problems had the highest prevalence (56.3\%). Hypertension had the second highest frequency (48.8\%). Circulatory diseases, cancer, arthritis and heart attack were other common problems with frequencies of $33.4 \%, 16.0 \%, 21.0 \%$ and $13.1 \%$, respectively. The possibility of having only one disorder was $22.2 \%$, while $14.5 \%$ of the 
individuals were not diagnosed with any of the conditions listed in the CMI. Participants who had two or more of these health problems constituted $63.3 \%$ of the cohort.

Each of these conditions were analyzed to search for their association with sleep efficiency latent classes (Table 4). The possibility of having breathing problems for each latent class decreased as sleep efficiency increased. The medium efficiency cluster had $6 \%$ lower prevalence and the high efficiency cluster had $16 \%$ lower prevalence compared to low efficiency cluster $\left(\mathrm{Chi}^{2}(2)=25.66, \mathrm{p}<0.001\right)$. A similar relationship was observed for hypertension and circulatory diseases. The likelihood of having circulatory problems was $14 \%$ lower $\left(\mathrm{Chi}^{2}(2)=25.05, \mathrm{p}<0.001\right)$ and the likelihood of having hypertension was $12 \%$ lower $\left(\mathrm{Chi}^{2}(2)=16.09, \mathrm{p}<0.001\right)$ in individuals belonging to the high efficiency cluster compared with those in the low efficiency cluster. In addition, there was a $13 \%$ higher prevalence of arthritis in those belonging to the low efficiency cluster compared to those in the high efficiency cluster $\left(\mathrm{Chi}^{2}(2)=29.83, \mathrm{p}<0.001\right)$. Finally, the number of comorbidities was higher in the low sleep efficiency cluster compared to high efficiency cluster $\left(\mathrm{Chi}^{2}(4)=17.79\right.$, $\mathrm{p}<0.001)$.

\section{DISCUSSION}

Poor sleep is one of the most important chronic health problems in modern societies with challenging working hours and artificial illumination being contributing factors(Medic et al., 2017; US Centers for Disease Control and Prevention, 2018). Understanding how sleep quality is influenced by social, environmental and biological factors may help to prevent health effects of poor sleep and generate novel strategies for better sleep hygiene practices. This research investigated three main areas: How sleep efficiency changes across older ages, whether there are subgroups of this longitudinal change and what the physical and mental health associations of worse sleep efficiency are. To answer these questions, we have investigated sleep characteristics in a longitudinal cohort of older adults followed for over 20 years. Using this UMLCHA data, individual trajectories in sleep efficiency not previously shown have been observed. Considering inter- and intra-individual variation, we 
showed that ageing is associated with a deterioration in sleep efficiency. However, the decline of sleep efficiency is not the same for each individual. Finally, we have shown that longitudinal low sleep efficiency with a steeper decline is associated with poor mental and physical health in the elderly. Hypertension, circulatory problems, arthritis and breathing problems were shown to be significantly associated with lower sleep efficiency latent class. Furthermore, this subgroup had a higher prevalence of depression recurrence.

Ageing can be considered as a time-related disruption of the physiology of organisms associated with a decline in health, function and survival. One widely reported association with ageing is low sleep efficiency. Sleep efficiency decline has been demonstrated longitudinally in a study group including 27 participants (age >75 years) whose baseline sleep characteristics were compared with 3-year follow-up(Hoch et al., 1997). They reported that the greatest change of sleep characteristics was in sleep efficiency. In our cohort, older participants had a shorter sleep length and a larger proportion of time spent in bed without sleeping, therefore reduced sleep efficiency. Results showed that the slope of sleep efficiency decline is $3.1 \%$, which is similar to previous findings(Ohayon et al., 2004). However, our longitudinal model had a slightly steeper slope compared to cross-sectional literature. Both cross-sectional literature and our longitudinal model predicted $90 \%$ sleep efficiency at age 40 but previous results estimated $75 \%$ sleep efficiency at age 100 compared to $70 \%$ in our model(Ohayon et al., 2004).

Many biological mechanisms have been proposed as underlying reason for these agerelated sleep changes. Firstly, sleep pressure increases as brain activity during the day increases. It has been shown that sensitivity of adenosine-dependent homeostatic sleep drive is decreased in older ages, which may influence continuity and duration(Mander et al., 2017). The other mechanism regulating sleep, the circadian rhythm, changes in older ages. The mammalian circadian rhythm pacemaker, the hypothalamic suprachiasmatic nucleus, differs in older ages in terms of cellular composition, neural activity and neurotransmission, which may alter synchrony amongst neurons, response to light, amplitude and period of the 
rhythm generated(De Nobrega \& Lyons, 2018; Duffy, Zitting, \& Chinoy, 2015). These may cause earlier sleep timing, increase the number of daytime naps and disrupt the continuity of night sleep. It has been shown that gender-specific biological and psychological factors may contribute ageing-related sleep changes(Lee et al., 2016; Mander et al., 2017). Females had lower sleep efficiency than males in our cohort. Female sex has been previously linked to low sleep quality(Fatima, Doi, Najman, \& Al Mamun, 2016) and steeper decline of sleep efficiency with ageing(Ohayon et al., 2004).

Social and environmental factors may also have an impact on sleep quality. Increased environmental disturbances such as noise generate more distraction for older individuals(Busby, Mercier, \& Pivik, 1994). Older people may be subject to more extrinsic effects on their sleep behavior that may include changes to their accommodation, retirement, bereavement, health decline and medication use(Rodda, Walker, \& Carter, 2011). Previous studies indicated that more physical activity increases sleep efficiency(Gubelmann, Heinzer, Haba-Rubio, Vollenweider, \& Marques-Vidal, 2018) and more outdoor daylight exposure entrains sleep timing(Roenneberg, Wirz-Justice, \& Merrow, 2003). Younger and working individuals may have more active life-style compared to older and non-working individuals and older adults may have reduced exposure to sunlight due to decreased outdoor activity. Our results are in line with this by showing an association between working and increased sleep efficiency.

The ageing world population is making age-related health problems a major public health issue. Worsening sleep as we age has been recognized as a contributor towards health problems in the elderly(De Nobrega \& Lyons, 2018). Predicted brain age using an electroencephalogram of sleep, has showed that people have healthier ageing if their estimated brain age is less than their chronological age(Sun et al., 2019). Finding the underlying reasons of good sleep in the elderly may help us to improve healthy ageing. Hypothesis-free, cluster analysis is being used to discover latent classes of sleep quality(Gadie et al., 2017; Yu, Mahendran, Abdullah, Kua, \& Feng, 2017). Gadie et al. in 
2017 using this approach showed that sleep quality, measured by the Pittsburgh Sleep Quality Index, can be explained by four subtypes that comprise 'good sleepers', 'inefficient sleepers', 'delayed sleepers' and 'poor sleepers'(Gadie et al., 2017). They reported that inefficient sleepers are more commonly found amongst older adults and the decline in sleep quality was best explained by a decrease in sleep efficiency(Gadie et al., 2017). We also used the latent class analysis, but instead of clustering sleep quality, we clustered the sleep efficiency criteria and its longitudinal change. In addition to the generalized features of ageing and low sleep efficiency, our research shows that there may be unexplained individual differences in terms of sleep variation with age.

We have identified a subgroup of older adults who seem more resistant to a decrease in sleep efficiency with ageing after adjusting for previously reported predictors of sleep behavior. The decline in sleep efficiency may be explained by both a decrease in sleep duration and increase in time spent in bed which may be a result of either increased sleep fragmentation or altered sleep timing. For all latent classes, awakening time did not differ with age. However, an earlier going to bed time was observed for all classes. Therefore, increased time spent in bed may explain the general decline in sleep efficiency but did not explain why the high efficiency cluster was more stable. Sleep duration did not exhibit a significant change with age for the high efficiency cluster, which may be the reason for the stability. In contrast, the high efficiency cluster had a steeper increase in their number of night wakes, which may indicate sleep duration is a better predictor of stable sleep efficiency than sleep fragmentation. Understanding individual age-related changes may offer new approaches to treat sleep problems more efficiently in older people.

We have shown that sleep is important for well-being. Our research observed a significant association between worse sleep efficiency and depression. Sleep and affective disorders are commonly diagnosed simultaneously(Germain \& Kupfer, 2008). Moreover, reciprocal effects of anti-depressants, sleep medications and light therapies indicate a strong relation between these disorders(Germain \& Kupfer, 2008). Circadian rhythm and emotion-related 
brain regions are anatomically connected(Ciarleglio, Resuehr, \& McMahon, 2011). Genetic studies have demonstrated that circadian rhythm genetic variants are associated with mood disorders(Liu \& Chung, 2015). Our longitudinal data indicated that sleep efficiency decline is also important and it covaries with the recurrent episodes of depression.

Health problems may be directly or indirectly associated with sleep efficiency. We have shown that participants, who rated themselves to have very good subjective health, sleep more efficient than those of who rated themselves as experiencing poor health. Arthritis, breathing and cardiovascular problems were observed to have the highest prevalences in those health conditions reported by the cohort and were associated with low sleep efficiency. Both respiratory and cardiovascular diseases have been shown to disrupt sleep possibly by increasing arousal(Bradley, 1993; Craig, Teets, Lehman, Chinchilli, \& Zwillich, 1998). Arthritis, which may increase alertness due to pain, has been also shown to influence sleep quality(Westhovens, Van Der Elst, Matthys, Tran, \& Gilloteau, 2014). Conversely, sleep deficiency may also affect health. US Centers for Disease Control and Prevention reported that inadequate sleep may cause diabetes, cardiovascular disease, obesity and depression(US Centers for Disease Control and Prevention, 2018). Plausible mechanisms for the effect may include altered immune response, enhanced stressors or reduced clearance of toxins. It has been shown that sleep disruption alters the inflammatory responses(Castanon-Cervantes et al., 2010; Medic et al., 2017) and clogging of blood vessels(McAlpine et al., 2019). In addition, sleep is responsible for the detoxification of the brain(Xie et al., 2013). A reduction in the clearance of harmful molecules because of lack of sleep may lead to impaired homeostasis. Reduced slow wave activity during non-REM sleep has been linked to Alzheimer's disease pathology(Lucey et al., 2019). It was shown that sleep protects neurons from oxidative stress and inadequate sleep increased mortality due to oxidative damage in an animal model(Hill et al., 2018).

We used self-reported sleep data, therefore reporting bias is a limitation. Objective polysomnography or wrist actigraphy studies may be better for measuring sleep parameters 
including REM - non-REM cycle. However, subjective measures are still useful to collect data over long study periods for large sample sizes. This has been validated by the high correlation between objective and subjective sleep measures which shows a high effectiveness of self-reported sleep timing and duration questionnaires(Santisteban, Brown, \& Gruber, 2018). Our sleep questions are not a part of a validated questionnaire and our constructed sleep efficiency variable has limitations because it did not include specific sleep onset latency and wake after sleep onset information. Nevertheless, the high correlation between PSQI variables and our derived sleep variables supports the validity of our measure for the study of sleep characteristics (Table S5). Another limitation is the usage of multiple depression scales. We standardized these measures, but this may still have a confounding effect. The UMLCHA cohort mostly comprised healthy individuals, thus disease groups had small sample sizes, which limited statistical power. Although the CMI has high correlation with clinical diagnosis,(Brodman et al., 1949) there may be a limitation with self-reporting error. In addition, it is not clear whether low sleep efficiency is causative or a manifestation of poor health. In addition, other covariates such as sedentary life style in older adults may have confounding effect in this association. Future studies with longitudinal cross-lagged panels may reveal the true causality between the comorbidities and low sleep efficiency. Although the sensitivity analysis indicated that the model successfully captured the longitudinal change, the results may be driven by both age categories and within subject follow-up because of the high number of missing data. Finally, non-random drop-out effects are problem for longitudinal old age studies as the drop-out participants tend to have poor health or higher risk of mortality(Rabbitt, Diggle, Holland, \& McInnes, 2004). Even though the joint model suggested that our model was robust, attrition may attenuate the observed decline of sleep quality in older ages.

\section{CONCLUSION}

Adequate sleep is important for better physical and mental health. To improve the quality of sleep, we must first understand how social and biological factors affect it. Longitudinal mixed- 
effect models enable us to account for within-individual variance of health and lifestyle and to investigate individual trajectories. Ageing was shown to be associated with decreasing sleep efficiency between the ages of 40 and 100. However, this decline was not universally observed. We have shown using latent class models that change of sleep efficiency can be separated into three clusters, in which one class was highly time-invariant with efficient sleep. This subgroup had a lower prevalence of cardiovascular and respiratory diseases and arthritis, along with a lower likelihood of depression recurrence. Investigating the genetic, biomedical and psychosocial reasons of this stability in individual sleep trajectories may pave the way for new directions to manage sleep disorders and to promote healthy ageing. 


\section{References}

Alden, D., Austin, C., \& Sturgeon, R. (1989). A correlation between the Geriatric Depression Scale long and short forms. Journal of Gerontology: Psychological Sciences, 44(4), $124-125$.

Anafi, R. C., Kayser, M. S., \& Raizen, D. M. (2019). Exploring phylogeny to find the function of sleep. Nature Reviews Neuroscience, 20(2), 109-116. https://doi.org/10.1038/s41583-018-0098-9

Beck, A. T., Steer, R. A., \& Carbin, M. G. (1988). Psychometric properties of the Beck Depression Inventory: Twenty-five years of evaluation. Clinical Psychology Review, 8(1), 77-100. https://doi.org/10.1016/0272-7358(88)90050-5

Bliwise, D. L., Ansari, F. P., Straight, L. B., \& Parker, K. P. (2005). Age changes in timing and 24-hour distribution of self-reported sleep. American Journal of Geriatric Psychiatry, 13(12), 1077-1082. https://doi.org/10.1097/00019442-200512000-00007

Bradley, T. D. (1993). Sleep disturbances in respiratory and cardiovascular disease. Journal of Psychosomatic Research, 37 Suppl 1, 13-7. Retrieved from http://www.ncbi.nlm.nih.gov/pubmed/8445582

Brodman, K., Erdmann, A., Lorge, I., Wolff, H., \& Broadbent, T. (1949). The Cornell Medical Index: an adjunct to medical interview. Journal of the American Medical Association, 140(6), 530-534. https://doi.org/10.1001/jama.1949.02900410026007

Busby, K. A., Mercier, L., \& Pivik, R. T. (1994). Ontogenetic variations in auditory arousal threshold during sleep. Psychophysiology, 31(2), 182-188. https://doi.org/10.1111/j.1469-8986.1994.tb01038.x

Castanon-Cervantes, O., Wu, M., Ehlen, J. C., Paul, K., Gamble, K. L., Johnson, R. L., ... Davidson, A. J. (2010). Dysregulation of inflammatory responses by chronic circadian disruption. The Journal of Immunology, 185, 5796-5805. 
https://doi.org/10.4049/jimmunol.1001026

Ciarleglio, C. M., Resuehr, H. E. S., \& McMahon, D. G. (2011). Interactions of the serotonin and circadian systems: nature and nurture in rhythms and blues. Neuroscience, 197, 816. https://doi.org/10.1016/j.neuroscience.2011.09.036

Craig, T. J., Teets, S., Lehman, E. B., Chinchilli, V. M., \& Zwillich, C. (1998). Nasal congestion secondary to allergic rhinitis as a cause of sleep disturbance and daytime fatigue and the response to topical nasal corticosteroids. The Journal of Allergy and Clinical Immunology, 101(5), 633-7. Retrieved from http://www.ncbi.nlm.nih.gov/pubmed/9600500

Dashti, H. S., Jones, S. E., Wood, A. R., Lane, J. M., van Hees, V. T., Wang, H., ... Saxena, R. (2019). Genome-wide association study identifies genetic loci for self-reported habitual sleep duration supported by accelerometer-derived estimates. Nature Communications, 10(1), 1100. https://doi.org/10.1038/s41467-019-08917-4

De Nobrega, A. K., \& Lyons, L. C. (2018). Aging and the clock: Perspective from flies to humans. European Journal of Neuroscience, 1-28. https://doi.org/10.1111/ejn.14176

Duffy, J. F., Zitting, K.-M., \& Chinoy, E. D. (2015). Aging and circadian rhythms. Sleep Medicine Clinics, 10(4), 423-434. https://doi.org/10.1016/j.jsmc.2015.08.002

Fatima, Y., Doi, S. A. R., Najman, J. M., \& Al Mamun, A. (2016). Exploring gender difference in sleep quality of young adults: Findings from a large population study. Clinical Medicine and Research, 14(3-4), 138-144. https://doi.org/10.3121/cmr.2016.1338

Gadie, A., Shafto, M., Leng, Y., Cam-CAN, \& Kievit, R. A. (2017). How are age-related differences in sleep quality associated with health outcomes? An epidemiological investigation in a UK cohort of 2406 adults. BMJ Open, 7(7), e014920. https://doi.org/10.1136/bmjopen-2016-014920

Germain, A., \& Kupfer, D. J. (2008). Circadian rhythm disturbances in depression. Human 
Psychopharmacology: Clinical and Experimental, 23, 571-585.

https://doi.org/10.1002/hup.964

Gubelmann, C., Heinzer, R., Haba-Rubio, J., Vollenweider, P., \& Marques-Vidal, P. (2018).

Physical activity is associated with higher sleep efficiency in the general population: the CoLaus study. Sleep, 41(7). https://doi.org/10.1093/sleep/zsy070

Hill, V. M., O'Connor, R. M., Sissoko, G. B., Irobunda, I. S., Leong, S., Canman, J. C., ... Shirasu-Hiza, M. (2018). A bidirectional relationship between sleep and oxidative stress in Drosophila. PLoS Biology, 16(7), e2005206. https://doi.org/10.1371/journal.pbio.2005206

Hoch, C. C., Dew, M. A., Reynolds, C. F., Buysse, D. J., Nowell, P. D., Monk, T. H., ... Kupfer, D. J. (1997). Longitudinal changes in diary- and laboratory-based sleep measures in healthy "old old" and "young old" subjects: a three-year follow-up. Sleep, 20(3), 192-202. Retrieved from http://www.ncbi.nlm.nih.gov/pubmed/9178915

Kripke, D. F., Garfinkel, L., Wingard, D. L., Klauber, M. R., \& Marler, M. R. (2002). Mortality associated with sleep duration and insomnia. Archives of General Psychiatry, 59(2), 131-136. https://doi.org/10.1001/archpsyc.59.2.131

Lane, J. M., Liang, J., Vlasac, I., Anderson, S. G., Bechtold, D. A., Bowden, J., ... Saxena, R. (2017). Genome-wide association analyses of sleep disturbance traits identify new loci and highlight shared genetics with neuropsychiatric and metabolic traits. Nature Genetics, 49(2), 274-281. https://doi.org/10.1038/ng.3749

Lassale, C., Batty, G. D., Steptoe, A., Cadar, D., Akbaraly, T. N., Kivimäki, M., \& Zaninotto, P. (2019). Association of 10-year C-reactive protein trajectories with markers of healthy aging: findings from the english longitudinal study of aging. The Journals of Gerontology, Series A: Biological Sciences and Medical Sciences, 74(2), 195-203. https://doi.org/10.1093/gerona/gly028 
Lee, C. Te, Chiang, Y. C., Huang, J. Y., Tantoh, D. M., Nfor, O. N., Lee, J. F., ... Liaw, Y. P. (2016). Incidence of Major Depressive Disorder: Variation by Age and Sex in LowIncome Individuals. Medicine (United States), 95(15), 1-5. https://doi.org/10.1097/MD.0000000000003110

Liu, C., \& Chung, M. (2015). Genetics and epigenetics of circadian rhythms and their potential roles in neuropsychiatric disorders. Neuroscience Bulletin, 31(1), 141-159. https://doi.org/10.1007/s12264-014-1495-3

Lucey, B. P., McCullough, A., Landsness, E. C., Toedebusch, C. D., McLeland, J. S., Zaza, A. M., ... Holtzman, D. M. (2019). Reduced non-rapid eye movement sleep is associated with tau pathology in early Alzheimer's disease. Science Translational Medicine, 11(474), eaau6550. https://doi.org/10.1126/scitranslmed.aau6550

Mander, B. A., Winer, J. R., \& Walker, M. P. (2017). Sleep and human aging. Neuron, 94(1), 19-36. https://doi.org/10.1016/j.neuron.2017.02.004

McAlpine, C. S., Kiss, M. G., Rattik, S., He, S., Vassalli, A., Valet, C., ... Swirski, F. K. (2019). Sleep modulates haematopoiesis and protects against atherosclerosis. Nature, 566, 383-387. https://doi.org/10.1038/s41586-019-0948-2

Medic, G., Wille, M., \& Hemels, M. E. H. (2017). Short- and long-term health consequences of sleep disruption. Nature and Science of Sleep, 9, 151-161. https://doi.org/10.2147/NSS.S134864

Ohayon, M. M., Carskadon, M. A., Guilleminault, C., \& Vitiello, M. V. (2004). Meta-analysis of quantitative sleep parameters from childhood to old age in healthy individuals: developing normative sleep values across the human lifespan. Sleep, 27(7), 12551273. https://doi.org/10.1093/sleep/27.7.1255

Rabbitt, P., Diggle, P., Holland, F., \& McInnes, L. (2004). Practice and drop-out effects during a 17-year longitudinal study of cognitive aging. The Journals of Gerontology: Series B, 
59(2), 84-97. https://doi.org/10.1093/geronb/59.2.P84

Rabbitt, P. M. A., McInnes, L., Diggle, P., Holland, F., Bent, N., Abson, V., ... Horan, M. (2004). The University of Manchester Longitudinal Study of Cognition in Normal Healthy Old Age, 1983 through 2003. Aging, Neuropsychology, and Cognition, 11(2-3), 245279. https://doi.org/10.1080/13825580490511116

Rodda, J., Walker, Z., \& Carter, J. (2011). Depression in older adults. BMJ, 343, d5219. https://doi.org/10.1136/bmj.d5219

Roenneberg, T., Wirz-Justice, A., \& Merrow, M. (2003). Life between clocks: daily temporal patterns of human chronotypes. Journal of Biological Rhythms, 18(1), 80-90. https://doi.org/10.1177/0748730402239679

Santisteban, J. A., Brown, T. G., \& Gruber, R. (2018). Association between the Munich Chronotype Questionnaire and wrist actigraphy. Sleep Disorders. https://doi.org/10.1155/2018/5646848

Sun, H., Paixao, L., Oliva, J. T., Goparaju, B., Carvalho, D. Z., van Leeuwen, K. G., ... Westover, M. B. (2019). Brain age from the electroencephalogram of sleep. Neurobiology of Aging, 74, 112-120. https://doi.org/10.1016/j.neurobiolaging.2018.10.016

Tampubolon, G., \& Maharani, A. (2018). Trajectories of allostatic load among older Americans and Britons: Iongitudinal cohort studies. BMC Geriatrics, 18, 255. https://doi.org/10.1186/s12877-018-0947-4

US Centers for Disease Control and Prevention. (2018). Sleep and sleep disorders. Retrieved January 28, 2019, from https://www.cdc.gov/sleep/index.html

Westhovens, R., Van Der Elst, K., Matthys, A., Tran, M., \& Gilloteau, I. (2014). Sleep problems in patients with rheumatoid arthritis. Journal of Rheumatology, 41(1), 31-40. https://doi.org/10.3899/jrheum.130430 
Xie, L., Kang, H., Xu, Q., Chen, M. J., Liao, Y., Thiyagarajan, M., ... Nedergaard, M. (2013). Sleep drives metabolite clearance from the adult brain. Science, 342(6156), 373-377. https://doi.org/10.1126/science.1241224

Yu, J., Mahendran, R., Abdullah, F. N. M., Kua, E.-H., \& Feng, L. (2017). Self-reported sleep problems among the elderly: A latent class analysis. Psychiatry Research, 258, 415420. https://doi.org/10.1016/j.psychres.2017.08.078 


\section{Figures and tables}

Figure 1: Timeline of the longitudinal study. Each bar represents a wave of a specific questionnaire, which is the period when a specific questionnaire is completed. Blue color is for sleep data that was collected with the Personal Details Questionnaire in 5 waves. Red color is for depression data in 11 waves (Beck Depression Inventory or Geriatric Depression Scale). Green color is for health data in 4 waves (Cornell Medical Index).

Figure 2: Changes in sleep efficiency with age. Blue dots show all available sleep efficiency data $(\mathrm{N}=10,062)$ for 5577 participants. The red line shows the predicted values across age in the mixed-effect linear model analysis after adjusting for gender, general health, social class and education level, smoking, alcohol intake, marital and employment status, usage of sleep medication. Grey area around the fit line shows 95\% confidence interval.

Figure 3: Change of sleep efficiency with age in different latent classes of sleep efficiency. Sleep efficiency clusters: Green dots (high efficiency, 1717 participants), red dots (medium efficiency, 2620 participants) and blue dots (low efficiency, 947 participants). The scatter plot shows all available sleep efficiency data for that cluster. Black lines show fit lines for each latent class with \%95 confidence interval.

Figure 4: Change of sleep duration (A), sleep fragmentation (B), getting up time (C) and going to bed time (D) with age in different latent classes of sleep efficiency. Sleep efficiency clusters: Green lines (high efficiency), red lines (medium efficiency) and blue lines (low efficiency). Fit lines for each latent class are shown with \%95 confidence interval.

Table 1: Descriptive statistics of time-variant and time-invariant predictors

Table 2: Sleep characteristics of the cohort

Table 3: Mixed-Effect Linear Model for Sleep Efficiency

Table 4: Disease prevalence in sleep efficiency latent classes

Table 1: Descriptive statistics of time-variant and time-invariant predictors 


\begin{tabular}{|c|c|c|c|c|c|}
\hline & wave-1 & wave-2 & wave-3 & wave-4 & wave-5 \\
\hline Total attendance & 6375 & 3176 & 624 & 751 & 575 \\
\hline Age (years) & $\begin{array}{l}\mathrm{N}=6355 \\
65.19 \pm 7.45\end{array}$ & $\begin{array}{l}\mathrm{N}=3150 \\
67.87 \pm 6.82\end{array}$ & $\begin{array}{l}\mathrm{N}=621 \\
76.23 \pm 5.70\end{array}$ & $\begin{array}{l}N=750 \\
80.56 \pm 5.34\end{array}$ & $\begin{array}{l}\mathrm{N}=572 \\
82.88 \pm 5.20\end{array}$ \\
\hline Marital status (\%) & $\mathrm{N}=5692$ & $\mathrm{~N}=2945$ & $\mathrm{~N}=579$ & $\mathrm{~N}=745$ & $\mathrm{~N}=570$ \\
\hline single, widowed, divorced, separated & 41.55 & 42.58 & 43.52 & 50.47 & 52.28 \\
\hline married, living with someone & 58.45 & 57.42 & 56.48 & 49.53 & 47.72 \\
\hline Working status (\%) & $\mathrm{N}=5651$ & $\mathrm{~N}=2935$ & $\mathrm{~N}=579$ & $\mathrm{~N}=743$ & $\mathrm{~N}=565$ \\
\hline unemployed, housewife, retired & 84.16 & 86.95 & 94.13 & 87.89 & 85.84 \\
\hline employed & 15.84 & 13.05 & 5.87 & 12.11 & 14.16 \\
\hline Smoking (\%) & $\mathrm{N}=5669$ & $\mathrm{~N}=2952$ & $\mathrm{~N}=574$ & $N=745$ & $N=564$ \\
\hline no & 82.22 & 85.37 & 94.60 & 96.64 & 96.10 \\
\hline yes & 17.78 & 14.63 & 5.40 & 3.36 & 3.90 \\
\hline Alcohol drinking (\%) & $\mathrm{N}=5444$ & $\mathrm{~N}=2903$ & $N=558$ & $N=735$ & $\mathrm{~N}=559$ \\
\hline no & 18.83 & 34.52 & 22.40 & 29.25 & 31.48 \\
\hline less than once in a day & 71.80 & 56.08 & 68.46 & 51.97 & 51.88 \\
\hline once in a day or more & 9.37 & 9.40 & 9.14 & 18.78 & 16.64 \\
\hline Current health (\%) & $\mathrm{N}=5659$ & $\mathrm{~N}=2899$ & $N=578$ & $N=734$ & $N=562$ \\
\hline very bad & 0.48 & 0.34 & 0.00 & 0.54 & 0.71 \\
\hline bad & 2.92 & 2.41 & 2.60 & 5.45 & 4.98 \\
\hline fair & 25.06 & 32.77 & 24.22 & 32.02 & 33.27 \\
\hline good & 46.69 & 47.60 & 53.98 & 47.14 & 42.88 \\
\hline very good & 24.86 & 16.87 & 19.20 & 14.85 & 18.15 \\
\hline Usage of sleep medication (\%) & $\mathrm{N}=5635$ & $\mathrm{~N}=2978$ & $\mathrm{~N}=575$ & $\mathrm{~N}=716$ & $\mathrm{~N}=558$ \\
\hline 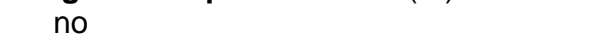 & 87.91 & 88.72 & 92.70 & 93.85 & 94.09 \\
\hline yes & 12.09 & 11.28 & 7.30 & 6.15 & 5.91 \\
\hline $\operatorname{Sex}(\%)$ & $\mathrm{N}=6371$ & & & & \\
\hline male & 30.09 & & & & \\
\hline female & 69.91 & & & & \\
\hline Social class (\%) & $\mathrm{N}=5893$ & & & & \\
\hline professional occupations & 4.90 & & & & \\
\hline intermediate occupations & 33.94 & & & & \\
\hline skilled non-manual occupations & 28.61 & & & & \\
\hline skilled manual occupations & 23.69 & & & & \\
\hline partly skilled occupations & 7.94 & & & & \\
\hline unskilled occupations & 0.92 & & & & \\
\hline Education level (\%) & $\mathrm{N}=5744$ & & & & \\
\hline pre-primary education & 0.00 & & & & \\
\hline primary education & 0.00 & & & & \\
\hline lower secondary education & 40.27 & & & & \\
\hline upper secondary education & 18.25 & & & & \\
\hline post-secondary non-tertiary education & 20.25 & & & & \\
\hline tertiary education first stage & 20.80 & & & & \\
\hline tertiary education second stage & 0.44 & & & & \\
\hline
\end{tabular}

Data collected using the Personal Details Questionnaire (PDQ) 
Table 2: Sleep characteristics of the cohort

\begin{tabular}{ccccccccccc}
\hline \multicolumn{4}{c}{ Sleep duration } & \multicolumn{2}{c}{$\begin{array}{l}\text { Number of night } \\
\text { wakes }\end{array}$} & \multicolumn{2}{c}{ Getting up time } & Going to bed time & Sleep efficiency \\
\hline Wave & $\mathrm{N}$ & $\begin{array}{c}\text { mean } \pm \text { SD } \\
(\mathrm{h})\end{array}$ & $\mathrm{N}$ & mean $\pm \mathrm{SD}$ & $\mathrm{N}$ & $\begin{array}{c}\text { mean } \pm S D \\
(\mathrm{hh}: \mathrm{mm})\end{array}$ & $\mathrm{N}$ & $\begin{array}{c}\text { mean } \pm \mathrm{SD} \\
(\mathrm{hh}: \mathrm{mm})\end{array}$ & $\mathrm{N}$ & $\begin{array}{c}\text { mean } \pm S D \\
(\%)\end{array}$ \\
\hline 1 & 5557 & $6.97 \pm 1.18$ & 5328 & $1.37 \pm 1.24$ & 5616 & $07: 51 \pm 00: 47$ & 5579 & $23: 15 \pm 00: 48$ & 5444 & $81.45 \pm 13.31$ \\
\hline 2 & 2925 & $6.89 \pm 1.22$ & 2806 & $1.44 \pm 1.21$ & 2978 & $07: 52 \pm 00: 47$ & 2936 & $23: 12 \pm 00: 49$ & 2870 & $80.12 \pm 13.79$ \\
\hline 3 & 571 & $6.86 \pm 1.21$ & 549 & $1.83 \pm 1.14$ & 575 & $07: 44 \pm 00: 47$ & 572 & $23: 02 \pm 00: 50$ & 561 & $79.34 \pm 13.59$ \\
\hline 4 & 693 & $6.57 \pm 1.24$ & $\mathrm{NA}$ & $\mathrm{NA}$ & 732 & $07: 51 \pm 00: 50$ & 733 & $22: 52 \pm 00: 50$ & 680 & $74.34 \pm 14.53$ \\
\hline 5 & 554 & $6.74 \pm 1.29$ & 484 & $2.23 \pm 1.03$ & 558 & $07: 47 \pm 00: 51$ & 556 & $22: 48 \pm 00: 52$ & 543 & $75.70 \pm 13.73$ \\
\hline
\end{tabular}

Sleep variables obtained from the Personal Detail Questionnaire (PDQ)

$\mathrm{N}$ : sample size, SD: standard deviation, h: hour, m: minute, NA: not available 
Table 3: Mixed-Effect Linear Model for Sleep Efficiency

\begin{tabular}{|c|c|}
\hline & Sleep efficiency \\
\hline Constant & $96.94[2.44]^{\star *}$ \\
\hline \multicolumn{2}{|l|}{ Gender } \\
\hline Age & $-0.31[0.01]^{\star \star}$ \\
\hline \multicolumn{2}{|l|}{ Social class (ref.: professional) } \\
\hline intermediate occupations & $0.40[0.80]$ \\
\hline skilled non-manual occupations & $0.37[0.90]$ \\
\hline skilled manual occupations & $1.31[0.90]$ \\
\hline partly skilled occupations & $1.38[1.07]$ \\
\hline unskilled occupations & $3.66[2.07]$ \\
\hline \multicolumn{2}{|l|}{ Education level (ref.: lower secondary) } \\
\hline upper-secondary education & $0.90[0.47]$ \\
\hline post-secondary non-tertiary education & $2.15[0.47]^{\star \star}$ \\
\hline tertiary education first stage & $2.08[0.55]^{\star *}$ \\
\hline tertiary education second stage & $1.62[2.42]$ \\
\hline \multicolumn{2}{|l|}{ Working status } \\
\hline occupied & $1.67[0.36]^{\star *}$ \\
\hline \multicolumn{2}{|l|}{ Marital status } \\
\hline married, living with someone & $0.40[0.32]$ \\
\hline \multicolumn{2}{|l|}{ Smoking } \\
\hline non-smoking & $-1.97[0.40]^{\star *}$ \\
\hline \multicolumn{2}{|l|}{ Alcohol usage (ref.: not drinking) } \\
\hline less than once in a day & $-0.80[0.31]^{*}$ \\
\hline once in a day or more & $-0.56[0.51]$ \\
\hline \multicolumn{2}{|l|}{ General health rating (ref.: very bad) } \\
\hline bad & $-1.57[2.01]$ \\
\hline fair & $1.79[1.93]$ \\
\hline good & $3.60[1.93]$ \\
\hline very good & $5.76[1.95]^{*}$ \\
\hline \multicolumn{2}{|l|}{ Usage of sleep medication } \\
\hline not using & $4.99[0.45]^{\star *}$ \\
\hline \multicolumn{2}{|c|}{$\begin{array}{l}\text { (Presented are regression coefficients and standard errors, ref.: } \\
\text { Reference variable) Sleep efficiency model: Mixed-effect maximum } \\
\text { likelihood regression, number of entity }=5284 \text {, number of cases }=9260 \text {, } \\
\text { Wald } x 2(21)=1357.57, p \text {-value }<0.001 \\
\left.\left.{ }^{*} \text { (regression } p \text {-value }<0.05\right),{ }^{* *} \text { (regression } p \text {-value }<0.001\right)\end{array}$} \\
\hline
\end{tabular}


Table 4: Disease prevalence in sleep efficiency latent classes

Sleep efficiency latent classes

p-value

Lower class Medium class High class

\begin{tabular}{lrrrc}
\hline Recurrent Depression & $40.35 \%$ & $21.85 \%$ & $16.37 \%$ & $<0.001$ \\
\hline Cornell Medical Index & & & & \\
\hline Hypertension & $56.04 \%$ & $49.28 \%$ & $44.12 \%$ & $<0.001$ \\
Heart attack & $14.25 \%$ & $11.78 \%$ & $13.67 \%$ & 0.28 \\
Circulatory Problems & $43.48 \%$ & $33.11 \%$ & $29.34 \%$ & $<0.001$ \\
Arthritis & $29.23 \%$ & $21.39 \%$ & $15.97 \%$ & $<0.001$ \\
Epilepsy & $2.42 \%$ & $2.65 \%$ & $2.29 \%$ & 0.87 \\
Stroke & $8.96 \%$ & $8.77 \%$ & $8.77 \%$ & 0.99 \\
Parkinson disease & $0.97 \%$ & $1.13 \%$ & $0.48 \%$ & 0.29 \\
Diabetes & $6.30 \%$ & $6.34 \%$ & $7.32 \%$ & 0.64 \\
Cancer & $16.30 \%$ & $16.48 \%$ & $15.85 \%$ & 0.93 \\
Breathing Problems & $65.01 \%$ & $58.86 \%$ & $49.38 \%$ & $<0.001$
\end{tabular}

CMI Comorbidities

$\begin{array}{lrrrr}\text { No comorbidity } & 3.38 \% & 5.57 \% & 6.64 \% & \\ \text { One comorbidity } & 6.76 \% & 8.63 \% & 8.97 \% & 0.001 \\ \text { Two or more comorbidities } & 89.86 \% & 85.80 \% & 84.39 \% & \end{array}$

Association analyses between patient health history and sleep efficiency latent clusters. Disease histories of participants were collected by the Cornell Medical index (CMI). Depression was measured by the Beck Depression Inventory (BDI) or the Geriatric Depression Scale (GDS) during the study period. The table shows the frequency of individuals with the health problem in each sleep efficiency latent class. Association analyses were performed using Pearson $\mathrm{chi}^{2}$ test and $\mathrm{p}$-values were reported in the table. 


\section{FIGURE 1}

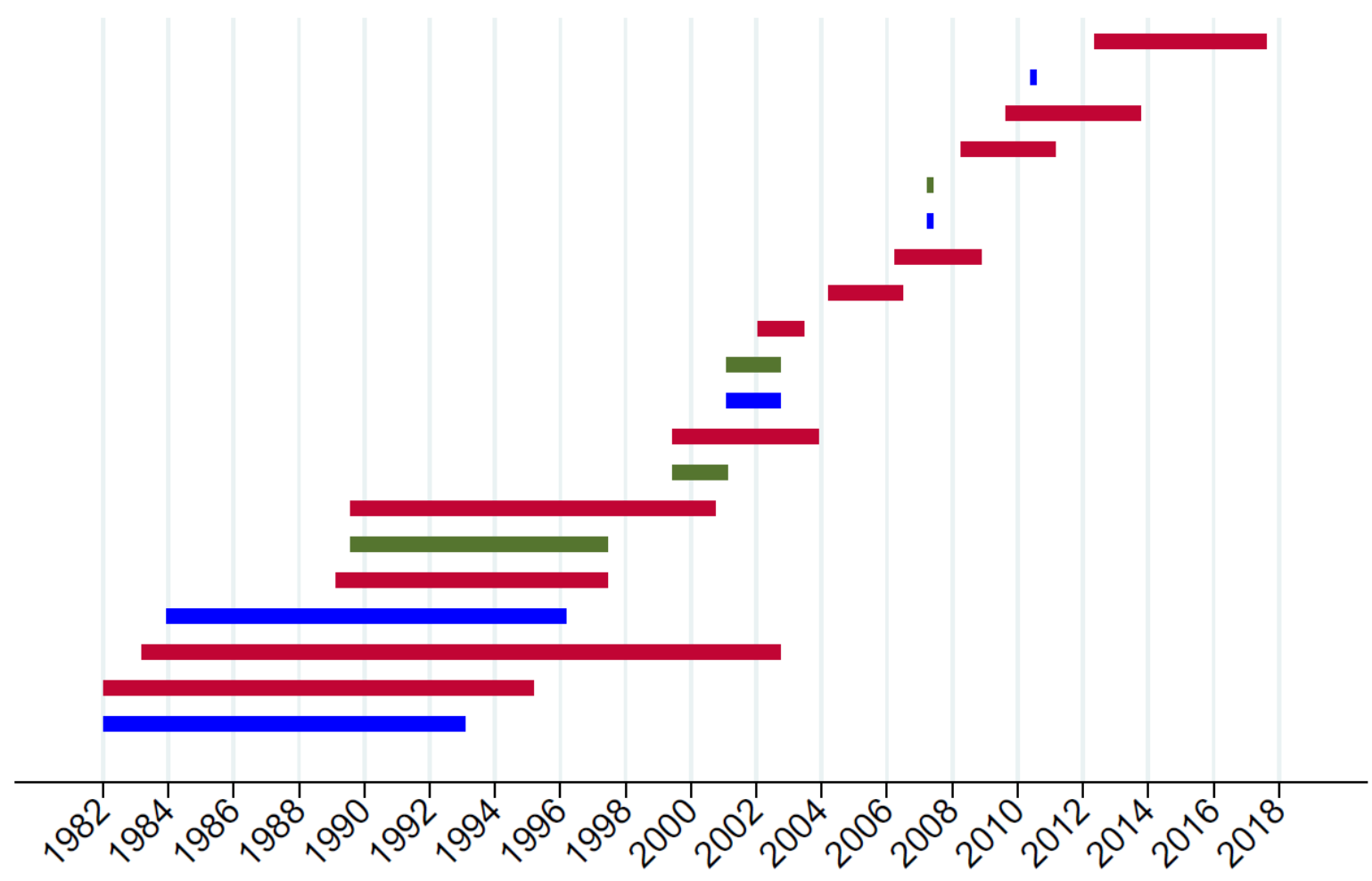

Sleep questionnaires $=$ Depression questionnaires $=$ Health questionnaires 
FIGURE 2

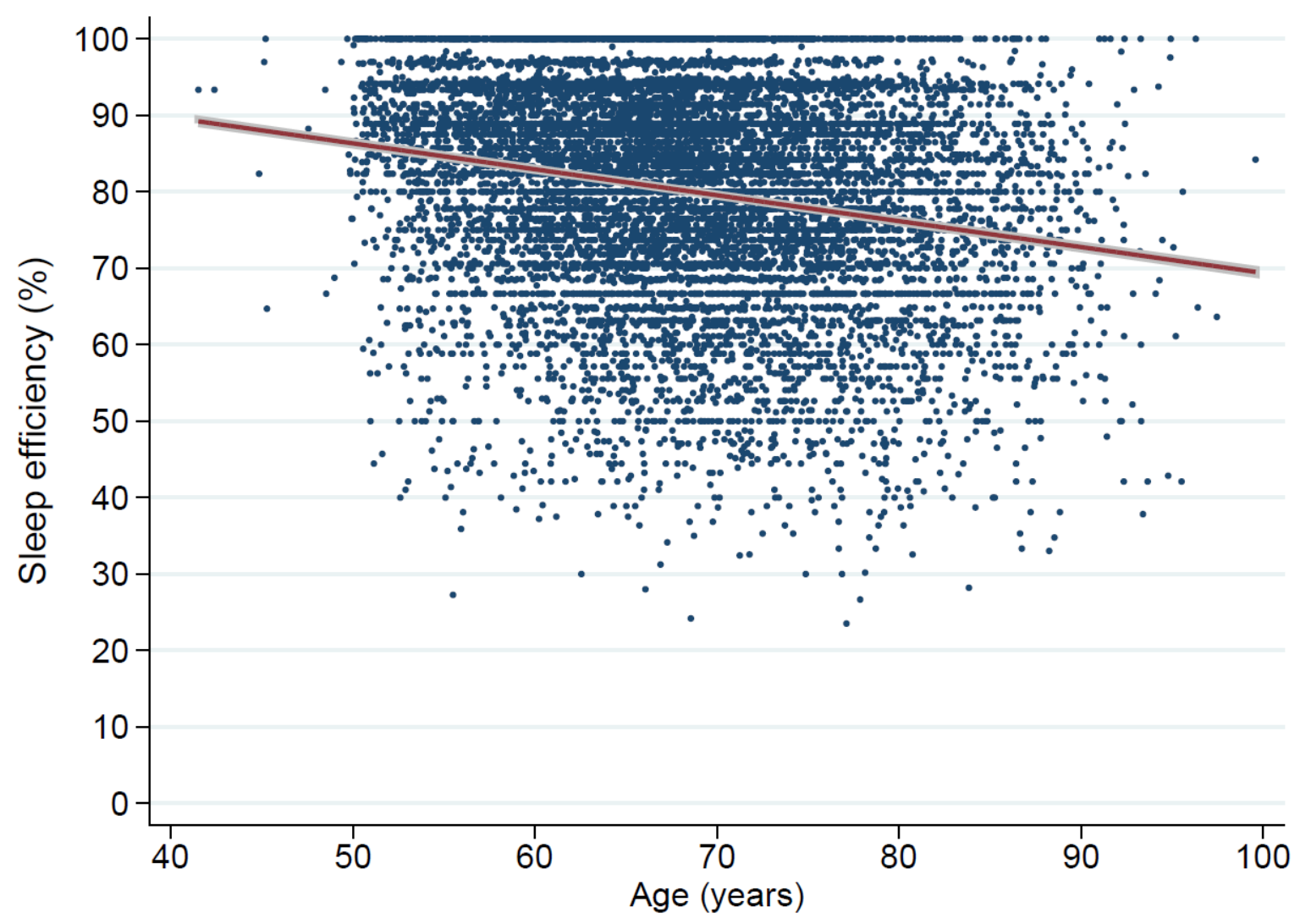


FIGURE 3

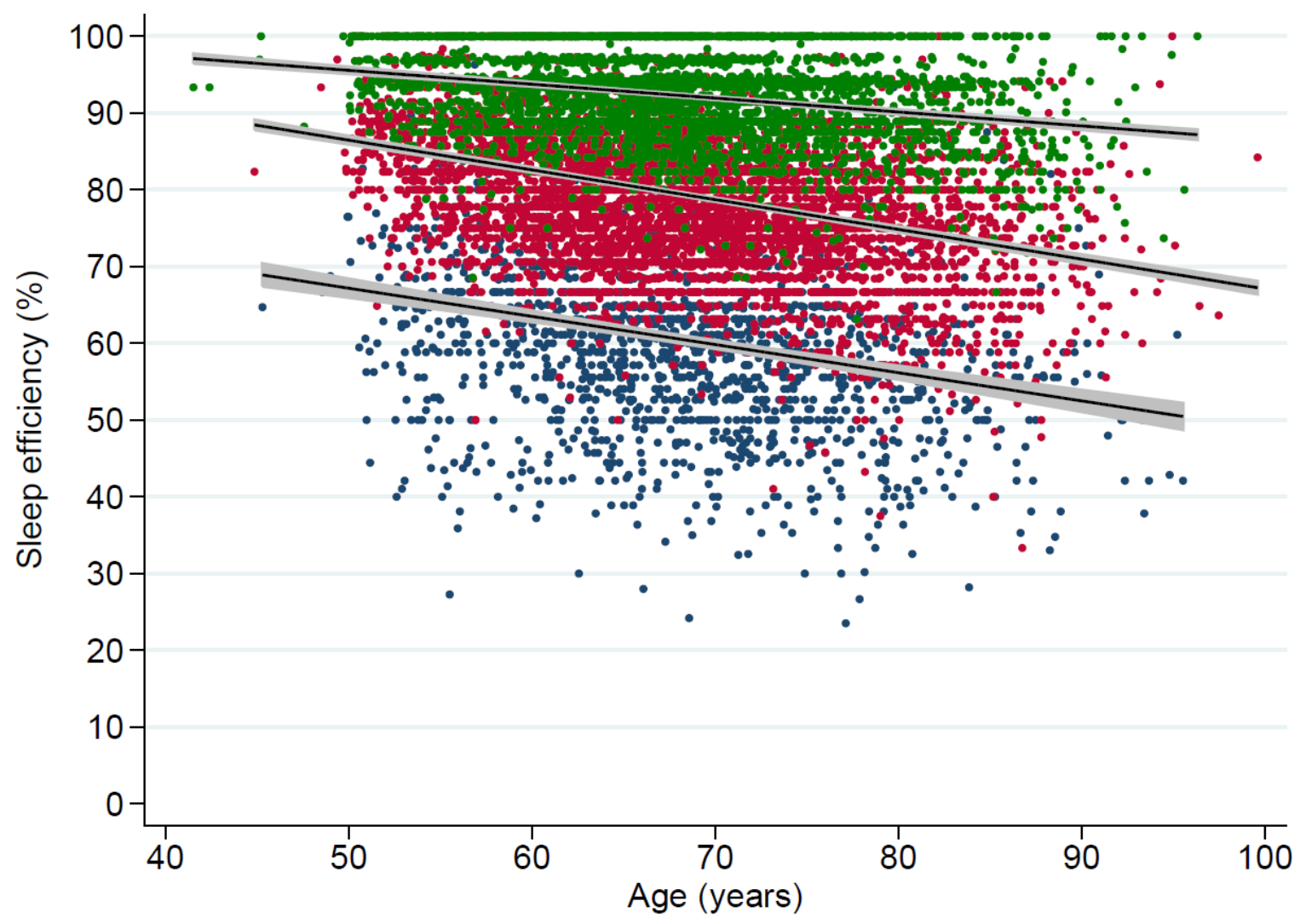




\section{FIGURE 4}
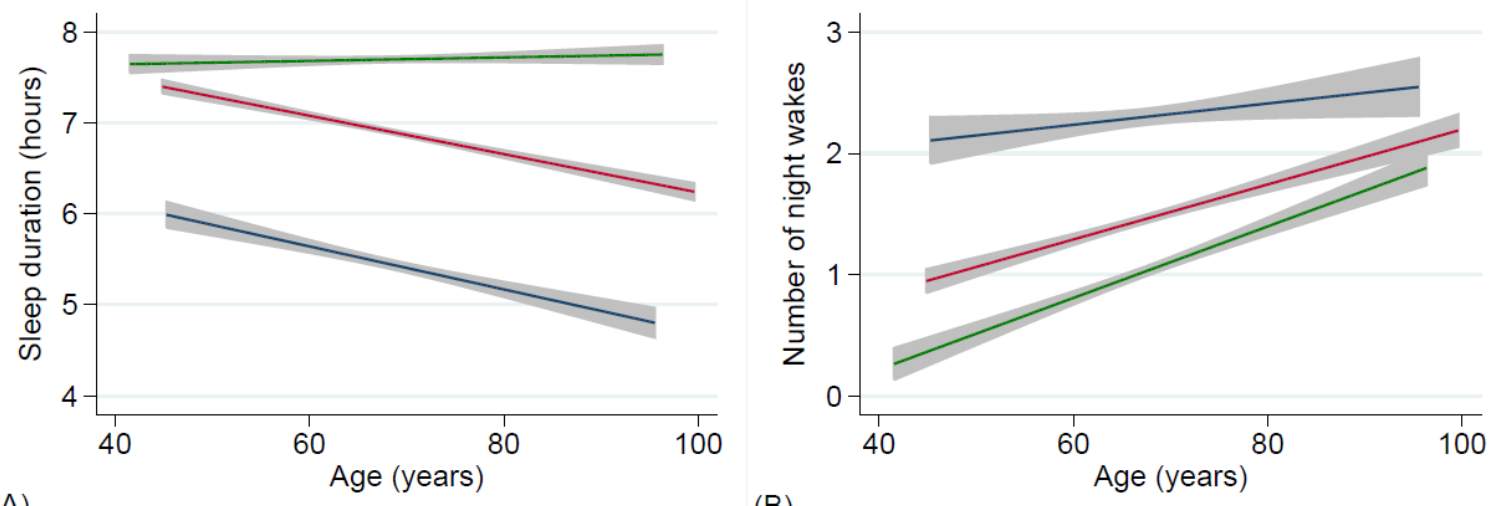

(A)

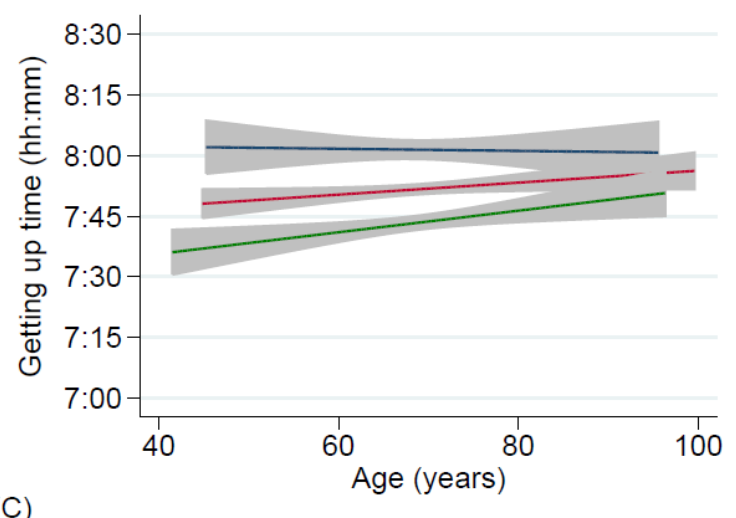

(C)
(B)

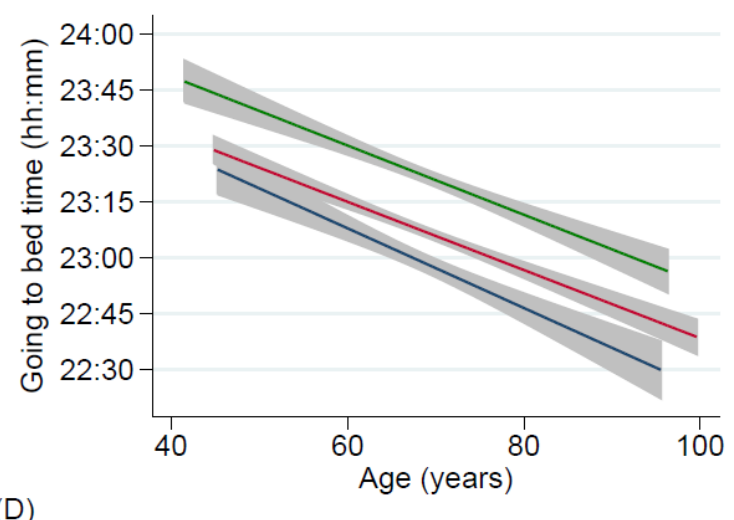

